\title{
Reflexivos e intensificadores en las formaciones con auto-: perspectiva histórica ${ }^{1}$
}

\author{
Verónica Orqueda* \\ Karem Squadrito* \\ Pontificia Universidad Católica de Chile
}

\begin{abstract}
Resumen
En este trabajo se analizan las formaciones reflexivas y no reflexivas, generalmente conocidas como de intensificación (König 2011), construidas con el prefijo auto- presentes en el Corpus Diacrónico del Español (CORDE). Nuestro objetivo es determinar si el autointensificador es diacrónicamente secundario con respecto al autoreflexivo. A partir de un enfoque tipológico funcional, en línea con las propuestas de Givón (2001), Haspelmath (2008) y König, Siemund y Töpper (2013), quienes avalan la polifuncionalidad semántica de los potenciales marcadores reflexivos, se clasificó y caracterizó tales formaciones según su función y categoría gramatical. La muestra resultante fue distribuida en periodos temporales. Como resultado, se observó no solo que los casos no reflexivos se encuentran desde los textos más antiguos a la par con los reflexivos, sino también que su
\end{abstract}

1 La presente investigación se enmarca en el proyecto CONICYT-FONDECYT 3150246 y constituye una reelaboración de una comunicación presentada en el Coloquio de Investigaciones en Gramática y Léxico (INCIHUSA, CCT, Mendoza, 16-17 de junio de 2016). Agradecemos a los participantes por todas las observaciones hechas en su momento.

* Para correspondencia, dirigirse a Verónica Orqueda (vorqueda@uc.cl) o a Karem Squdrito (krsquadrito@uc.cl); Departamento de Lingüística Facultad de Letras, Pontificia Universidad Católica de Chile. Avenida Vicuña Mackenna \#4860, Santiago de Chile. 
crecimiento es sostenido a lo largo del tiempo. Si bien la cantidad total de reflexivos supera a los restantes en todos los periodos temporales analizados, se concluye que las formaciones con auto- intensificador no deben considerarse necesariamente como secundarias desde un punto de vista diacrónico.

Palabras clave: formación de palabras, reflexivos, intensificadores, cambio diacrónico.

\title{
REFLEXIVES AND INTENSIFIERS IN WORD-FORMATIONS WITH AUTO-: A HISTORICAL PERSPECTIVE
}

\begin{abstract}
In this paper, we analyze reflexive and non-reflexive formations with the prefix auto- (the latter better known as "intensifiers" [König, 2011]) in the Diachronic Corpus of Spanish (referred to as CORDE). The aim is to determine whether the use of this prefix as an intensifier is diachronically secondary to its use as a reflexive.From a typological functional approach, in line with works such as Givón (2001), Haspelmath (2008) and König, Siemund and Töpper (2013), which support the semantic polyfunctionality of potential reflexive markers, we classified and characterized such formations according to their function and grammatical class, and distributed the sample into different time spans. As a result, we observed not only that nonreflexive cases with auto- are present already in the earliest texts (as reflexives are), but also that their use grows steadily through time. We conclude that, although the whole number of reflexives is larger than that of non-reflexives at all stages, formations with the intensifying auto- are not necessarily secondary from a diachronic perspective.
\end{abstract}

Keywords: Word-formation, reflexives, intensifiers, diachronic change.

Recibido: 21/12/16 Aceptado: 07/09/17

\section{INTRODUCCIÓN}

Sin duda, uno de los campos más ricos para el estudio de la interrelación entre morfosintaxis y semántica se encuentra en el fenómeno de formación de palabras, ya sea a través de procesos como afijación (prefijación y sufijación), ya de composición. Un caso particular en el que se observa esta relación 
es el de la formación de palabras a partir de estrategias de reflexividad y de otros valores asociados, como la focalización y la anticausatividad. Así por ejemplo, el prefijo de origen griego auto- en español no solo da lugar a formaciones reflexivas como en autocrítica, autoanálisis y autofotografia $\left(\right.$ auto- $\left.{ }_{1}\right)$, sino que también otorga otros posibles valores: de focalización como en autoaprendizaje (auto- ${ }_{2}$ ) y de espontaneidad o anticausatividad del evento como en autoaplicador y autodisparador (auto- ${ }_{3}$ ).

En torno a la distinción entre estos diferentes valores existen varios trabajos, especialmente los de Felíu $(2003,2005)$ entre los más exhaustivos, y el de Mutz (2003) cuyas conclusiones, aunque centradas en el italiano, pueden extenderse en cierta medida a otras lenguas romances. Mientras que estos trabajos adoptan una perspectiva sincrónica, por nuestra parte creemos que la incorporación del aspecto diacrónico también puede aportar datos necesarios para entender el fenómeno de manera global. De allí que nuestra aportación pretenda ser, precisamente, el análisis diacrónico no con el fin de aceptar o rechazar las propuestas existentes sino fundamentalmente de complementarlas. Creemos que, en particular, un acercamiento diacrónico puede contribuir a la consideración de los diferentes valores de auto- como primarios y secundarios.

Así, mediante un análisis exhaustivo y la clasificación de las formaciones reflexivas y de intensificación con el prefijo auto- a lo largo de la historia del español, intentaremos establecer si es posible asociar uno de los valores del prefijo (específicamente, reflexivo o no reflexivo) a un rasgo secundario con respecto al otro; $y$, en segundo lugar, en el eventual caso de un cambio diacrónico, comparar su direccionalidad con aquella otra observable en la formación de cláusulas verbales con valores reflexivos y de intensificación, extensamente estudiados en la literatura. Tal comparación nos permitirá observar que los fenómenos encontrados en el ámbito de la formación de palabras sirven para comprender la formación de cláusulas y viceversa.

En los trabajos en torno a este prefijo encontramos que sus diferentes usos, reflexivos y no reflexivos, se diferencian entre primarios y secundarios. Así, por ejemplo, Felíu (2003: 53) es explícita en el rasgo secundario de los no reflexivos:

Junto a este uso reflexivo de auto-, que consideramos primario ya que se documenta en la mayor parte de las piezas léxicas formadas con este prefijo, existe también un uso secundario no reflexivo, que denominaremos "modal enfático". En las formaciones en las que el prefijo auto- presenta este segundo valor no existe una relación de reflexividad, sino que se resalta la ausencia de una causa externa al evento (...) 
Si pensamos en tres maneras en que puede entenderse el posible carácter secundario de este valor, es decir, a) en términos estadísticos (al comparar la cantidad de casos de uno y otro tipo), b) en términos cognitivos (según la percepción de los propios hablantes) y c) en términos diacrónicos (considerando el eventual desarrollo histórico de uno a partir del otro), es evidente que la afirmación de Felíu se relaciona con el primer tipo de acercamiento. Ahora bien, la posibilidad de un carácter secundario desde una de estas perspectivas nos permite preguntarnos si lo mismo ocurre a nivel diacrónico. Por esto, uno de nuestros objetivos consiste precisamente en buscar argumentos a favor o en contra de esta afirmación. Así, en este trabajo nos centraremos en el último tipo de acercamiento, el diacrónico, con el fin de complementar los datos existentes, especialmente relativos a la cuantificación de datos en corpus del español. Como es de suponer, partiremos con la hipótesis de que no es posible que diacrónicamente el uso modal enfático sea secundario con respecto al reflexivo, dado que lo esperable es que en el ámbito de formación de palabras la dirección de cambio sea la misma que encontramos en el ámbito oracional en diferentes lenguas del mundo: de intensificador a reflexivo (König y Siemund 2000, Heine y Kuteva 2002, Heine 2005, entre otros).

\section{ANTECEDENTES Y MARCO TEÓRICO}

\subsection{ANTECEDENTES: LAS FORMACIONES CON AUTO- EN ESPAÑOL}

Desde un punto de vista morfológico, las formaciones con auto- se distribuyen en tres grupos diferentes, tal como sistematiza Felíu (2003): a) aquellas en las que auto- se considera un lexema, ya sea por el desarrollo regular de la antigua forma acto, ya por el truncamiento a partir de automóvil, tal como ocurre en autoescuela o autovía (Varela 2005), b) aquellas en las que auto-, en tanto tema, es capaz de recibir sufijos, como en autismo, autofagia; y c) aquellas en las que se trata de un verdadero prefijo derivativo, como en autopercepción, autobiografía, capaz de unirse a un elemento léxico preexistente en la lengua, sea o no de procedencia culta. Así, mientras que algunos autores agrupan todos los casos bajo la noción de prefijoide debido a su origen culto (Lang 1990, por ejemplo), parece evidente que se pueden diferenciar tres procesos diferentes: composición, derivación culta o derivación simple, según el tipo de formación. Nótese también que una clasificación particular es la que propone García-Medall (1993), para quien 
se trata de un fenómeno de truncamiento, pero no a partir de automóvil sino de automático, de lo cual resultaría que formaciones del tipo autopiloto, autoalarma (auto-X) serían equivalentes a piloto automático, alarma automática (X + automático), con las cuales coexistirían. Sin embargo, esto parece difícil de sostener ya que hay casos en los que no se conserva el mismo sentido en la paráfrasis. Así, por ejemplo, fuera de un contexto apropiado no es aceptable que autoenfocar equivalga en todos los casos a enfocar automáticamente, o que autolucidez equivalga a lucidez automática.

Sin duda, la diferenciación entre casos de prefijación y composición tiene un fuerte correlato en la productividad: los primeros, que son los que analizaremos en este trabajo, son mucho más productivos que los restantes, dando lugar en la actualidad a la formación tanto de sustantivos, como de adjetivos, verbos y de manera secundaria también de algunos adverbios, razón por la cual hablamos de formaciones y no estrictamente de nominalizaciones.

Desde un punto de vista sintáctico, se trata de un prefijo peculiar ya que, al igual que inter-/entre- y co-, tiene incidencia argumental (RAE 2010) incluso en casos en que no se trata de formaciones deverbativas. Como señala Felíu (2003), formaciones como autogol o autobiografía, aunque no necesariamente reproducen la estructura argumental de la forma base (compárese con biografía y autobiografía), sí presentan una en la forma resultante.

Por último, desde un punto de vista semántico existe menos consenso respecto de la posible clasificación de los casos de prefijación: mientras que en algunos trabajos se menciona únicamente el valor reflexivo (Varela 2005), en otros se hace hincapié en la posible distinción entre acciones o eventos con y sin el rasgo de voluntariedad, en la espontaneidad o la no intencionalidad de la acción (RAE 2010), o incluso en la consideración de este prefijo como uno de intensificación (Lang 1990). Por su parte, otros autores como Bosque y Demonte (1999) consideran el posible valor no reflexivo de este prefijo, utilizado para destacar la ausencia de una causa o agente externo a la acción como en ese metal se autodegrada, aunque para ellos funcionaría únicamente en la unión con ciertas bases verbales.

Por otra parte, tampoco hay consenso absoluto con respecto a si el valor reflexivo lo aporta el prefijo o si se trata del significado preexiste en el elemento al que se anexa auto- (Lang 1990; RAE 2010). En relación con este punto, es fundamental que la contribución de Felíu (2003), quien señala que no es necesario asumir la preexistencia de un valor reflexivo, tal como queda claro con formaciones como autogol o autobiografía. 


\subsection{REFLEXIVIDAD E INTENSIFICACIÓN DESDE UN MARCO TIPOLÓGICO-FUNCIONAL}

En línea con trabajos como los de Givón (2001) y Haspelmath (2008), consideramos los diferentes valores de este elemento como funciones esencialmente semánticas con un correlato en su funcionamiento sintáctico. Esta distinción es la que permite explicar la posible existencia de comportamientos variados, a diferencia de un modelo estrictamente funcional.

Dentro de este marco teórico, existe una importante línea de trabajos en los que se relacionan diacrónica y sincrónicamente los valores reflexivo y de intensificación en cláusulas verbales. Como se puede ver con el caso del inglés (y parcialmente con el español) a continuación, es frecuente que los valores semánticos puedan ser diferenciados a pesar de tratarse de marcadores formalmente idénticos:

(1) Cláusulas verbales (inglés/español)

(a) John criticized himself (c) 'John se criticó a sí mismo'

(b) John did it himself

(d) 'John lo hizo por sí mismo'

Mientras que himself tienen un valor esencialmente reflexivo y de correferencia en (1a), en (1b) funciona como foco contrastivo, atribuyendo la acción a John en oposición a otros eventuales referentes. De manera similar, en los casos de formación de palabras, self- puede indicar ambos valores, tal como ocurre con auto- en español:

(2) Formación de palabras (self-/auto-)

(a) self-destructive 'autodestructivo'(que se destruye a sí (c) autodestrucción mismo)

(b) self-made 'hecho por uno mismo' (*a sí mismo')

(d) autogestión

Mientras que el valor reflexivo es entendido como la correferencialidad de dos argumentos, típica pero no necesariamente Actor y Padecedor, considerando estos últimos como los esquemas de macrorroles en la propuesta de Van Valin (2001), el de intensificación ${ }^{2}$ se entiende en este marco teórico como aquellas situaciones en las que un constituyente (perteneciente a cualquier

\footnotetext{
2 Aunque coincidimos con varios especialistas (especialmente, los asistentes al Coloquio de Inv. en Gramática y Léxico) en lo poco apropiado que puede resultar el término "intensificación" y la posible confusión con otros tipos de elementos, hemos decidido mantener
} 
clase de palabra) prosódicamente prominente sirve para evocar posibles alternativas para el referente del sintagma nominal con el que se relaciona:

Reflexive pronouns (or "reflexive anaphors") are expressions which are prototypically used to indicate that a non-subject argument of a transitive predicate is coreferential with (or bound by) the subject, i.e. expressions like German sich, Russian sebja, Turkish kendi, Mandarin zijjŭ, English $x$-self (König, Siemund y Töpper 2013).

By intensifiers we mean expressions like German selbst, Russian sam, Turkish kendi, Mandarin zijĭ, English $x$-self, which can be adjoined to either NPs or VPs, are invariably focused and thus are prosodically prominent. The main function of intensifiers can be seen in the evoking of alternatives to the referent of the NP they relate to (König, Siemund y Töpper 2013).

Nótese que en el caso de cláusulas verbales del español, el fenómeno es bastante complejo debido a la presencia del se:

(3a) Reflexivo: Juan se vio en el espejo (a sí mismo)

(3b) Intensificador: El presidente mismo preparó el discurso

Así, mientras que mismo es el marcador de intensificación por excelencia, como reflexivo funciona únicamente como reforzador, ya que es posible indicar la correferencialidad únicamente mediante la marca verbal ${ }^{3}$.

En el ámbito de la formación de palabras en español, entenderemos como reflexivas las formaciones del tipo autodestrucción en (2c), por su posibilidad de paráfrasis como en "destrucción de sí mismo". Respecto de las formaciones no reflexivas, mantendremos la categoría de "intensificadores" para todo el conjunto, aunque admitiremos que se trata de una clasificación que alberga fenómenos diversos, anticausativos, focalizadores o atributivos, dependiendo esencialmente del contexto, de la clase de palabra y del tipo de derivación, entre otros factores (Felíu 2003), como se ejemplifica a continuación:

la terminología puesto que constituye un área ampliamente desarrollada previamente a nivel internacional.

Hemos simplificado aquí las cuestiones relativas a los valores asociados al se, puesto que no es un tema que se relacione de manera completamente directa. Para una revisión de los problemas asociados a este elemento, especialmente los valores medios y no reflexivos, véanse los trabajos de Maldonado (1999) y González (2006). 
(4a) [...] empapada de fuerte sentimiento de responsabilidad y autoacusación (CORDE 1936).

(4b) El uso de hornos metálicos, autocarbonizadores con recuperación de alquitrán (CORDE 1951).

(4c) [...] el hombre que ha cumplido en la historia la plenitud de su destino espiritual y que encuentra en la autoconciencia de este desarrollo (CORDE 1950).

(4d) [...] "Reinaldo Solar" recoge no pocos rasgos autobiográficos de su época de estudiante y de integrante de aquel idealista grupo de "La Alborada" (CORDE 1966).

Como se puede ver, mientras que en (4a) se trata de un sustantivo biargumental donde ambos participantes, Actor y Padecedor, son correferentes, en (4b) autocarbonizador hace referencia a la espontaneidad y la no intervención de un agente o una fuerza, por lo que puede entenderse como un anticausativo; por su parte, la presencia en (4c) de un complemento no correferente con el argumento externo lleva a ver este caso como uno de focalización, en tanto es el argumento externo (sujeto) el que aparece en un foco contrastivo con respecto a otros eventuales referentes; por último, en (4d) también se explicita un complemento interno, pero a diferencia del anterior, puede considerarse como parte del ámbito del argumento externo y es parafraseable por "su propio/a X", por lo que puede verse como un caso atributivo. Estos últimos, aunque mucho más extraños y menos frecuentes que el resto, pueden considerarse como casos a medio camino entre los valores no reflexivos y los de focalización.

Es importante señalar que esta distinción no aparece en ninguno de los autores consultados para el español. Aun así, parece funcionar dentro de la muestra como una distinción no solo posible sino también necesaria, más allá de un alto número de casos ambiguos, como es esperable, ya que es la mejor manera de explicar los posibles cambios. Si bien fue parte de la metodología de esta investigación el análisis discreto de todas las categorías, para los fines de este artículo las englobaremos de manera general como intensificadores, en tanto comparten el rasgo común de focalizar alguno de los elementos de la estructura argumental sin la necesidad de especificar la correferencialidad. Esta agrupación, por otra parte, busca seguir la intuición de los trabajos previos ya mencionados que acuden al posible rasgo secundario de estas formaciones, para poder aceptar o rechazar tal afirmación. 


\subsection{REFLEXIVIDAD E INTENSIFICACIÓN DESDE UNA PERSPECTIVA DIACRÓNICA}

En el ámbito de la formación de cláusulas verbales, ha recibido bastante atención el proceso translingüístico por el cual los marcadores reflexivos pueden originarse históricamente a partir de diferentes fuentes (Faltz 1985; König y Siemund 2000; Schladt 2000; Heine 2005), entre las cuales suele estar (o al menos ser una etapa intermedia) el de los marcadores de intensificación. Esta dirección de cambio que no se restringe a algunas familias lingüísticas en particular, se suele demostrar con el desarrollo de self en inglés (que pasa a ser parte del reflexivo por excelencia en inglés moderno mientras que simplemente enfatizaba la correferencia en inglés medieval), nafs 'alma' en árabe (nafs-ii 'mi alma'> 'mí mismo') y casos equivalentes en ciertos creoles (véase Heine 2005). De allí que sea válido como hipótesis la posibilidad de que la misma dirección de cambio se produzca en los casos de formación de palabras. Lamentablemente, la mayoría de los trabajos en torno a nominalizaciones no incluyen este tipo de fenómenos; en general esto se debe a que prefijos como auto- no son en sí mismos prefijos nominalizadores. De las pocas investigaciones que hay (König 2011) se podría inferir que un desarrollo similar no resultaría extraño.

\section{METODOLOGÍA}

La muestra en la que se basa la presente investigación está realizada partir del Corpus Diacrónico del Español (CORDE) ${ }^{4}$ y consiste en la totalidad de los datos encontrados en los que auto- funciona como un prefijo derivativo productivo. Es decir, se exceptúan los casos en que auto- es: (a) un lexema en sí mismo o un compuesto por acortamiento (auto sacramental, autopista) y (b) un tema culto (autofagia, autocracia, autógrafo). Por otro lado, con

4 El CORDE consiste en una recopilación de 125.000 .000 de palabras (74\% peninsular y $26 \%$ de otros orígenes) que comienza, en palabras de la RAE, desde los inicios del idioma y que se extiende hasta 1975. Si bien varios de los criterios de distribución de los datos son bastante cuestionables, sigue siendo el corpus más rico en cuanto a cantidades absolutas. Para efectos de este estudio, y debido a la diversidad de criterios de clasificación, la muestra de análisis está formada únicamente a partir del CORDE y no del CREA para no crear problemas metodológicos. Creemos que si bien los datos del CREA pueden contribuir de manera secundaria a las conclusiones de este trabajo, no las determinan ni las alteran. 
el fin de delimitar la muestra, se consideraron únicamente las formaciones sin guion (autogeneración vs. auto-generación).

La muestra obtenida consiste en un total de 1606 casos válidos, distribuidos entre los años 1589 (fecha del primer ejemplo: autobiografía) y 1975, los cuales fueron clasificados semánticamente y según clases de palabras (V, S, ADJ, ADV) y agrupados en tres períodos de 25 años, más un primer período más amplio pero con un número de datos mucho menos significativo, que abarca todos los casos anteriores al siglo $\mathrm{XX}^{5}$.

Para la determinación de frecuencias de uso, se ha preferido tener en cuenta el número de textos en los que una determinada forma aparece al menos una vez con un determinado valor, más que el número absoluto de casos. Así, por ejemplo, en casos en que una palabra pudiera aparecer utilizada 34 veces en total, pero en un solo texto, se contabilizó como 1 y no como 34, pues consideramos que de esta manera los resultados pueden ser más objetivos.

\section{RESULTADOS}

De los 1606 casos totales se analizaron 887, que corresponden al total de formaciones que aparecen al menos una vez en un texto. Estos, clasificados según los períodos temporales mencionados, se sistematizan de la siguiente forma:

\begin{tabular}{|l|c|c|c|}
\hline \multicolumn{1}{|c|}{ Valor } & Reflexivo & Intensificador & Totales \\
\hline $1589-1900$ & 25 & 8 & 33 \\
\hline $1901-1925$ & 73 & 21 & 94 \\
\hline $1926-1950$ & 155 & 76 & 231 \\
\hline $1951-1975$ & 327 & 202 & 529 \\
\hline Totales & 580 & 307 & 887 \\
\hline
\end{tabular}

Tabla 1: Distribución diacrónica de valores según la cantidad de textos

5 Si bien de esto se podría inferir que el prefijo se vuelve realmente productivo en el último siglo, esta afirmación debe tomarse con cautela ya que no contamos con la misma cantidad de textos de períodos anteriores y estos son esencialmente escritos, mientras que el uso del prefijo parecería estar más relacionado con la oralidad. 
Algunos ejemplos:

(5) Periodo 1:

(a) Refl.: autobiográfico, autobiografía, autosugestión, autorredención.

(b) Int.: autográfico, autolitográfico, autobiografía ${ }^{6}$ autografomanía, autodeclarar.

(6) Periodo 2:

(a) Refl.: autoanálisis, autocrítica, autodisciplina, autorrevelación, autosugestionar.

(b) Int:: autoclave, autoinducción, autodestruido, autoexcitatriz, autogenético.

(7) Periodo 3:

(a) Refl.: autobombo, autocastigo, autoconstituirse, autocontrol, autodivinizarse.

(b) Int.: autoafirmarse, autoametralladora, autoclave, autoerótico, autoestablecer.

(8) Periodo 4:

(a) Refl.: autoconvencerse, autocrítica, autodenominarse, autoelogio, autorreproche, autotitulado.

(b) Int.: autodeterminado, autodigestión, autoinjerto, autoinquisidor, autorreflexivo, autoungir.

Como se puede ver, el primer periodo no se puede considerar estadísticamente significativo pero aun así permite poner en evidencia que los primeros testimonios del español en los que se utiliza el prefijo auto- no son únicamente reflexivos. Con respecto a las cantidades absolutas, si se compara el total de reflexivos (643) con el de intensificadores (301), es evidente la diferencia y se podría hablar de un valor predominante y otro secundario. Sin embargo, a lo largo del desarrollo del morfema auto- en español es posible apreciar un crecimiento continuo de ambos valores semánticos; de hecho, desde el siglo XIX en adelante el crecimiento se desarrolla de manera exponencial y sostenida a lo largo de los documentos:

\footnotetext{
6 Nótese que, a diferencia de los trabajos previos sobre el tema, hemos considerado el contexto como herramienta para determinar el valor en cada caso. Por ejemplo, en el caso de "Son, en fin, la autobiografia de un alma ardiente, voluntariosa..." (CORDE 1823), la presencia del complemento lleva a analizar este caso como uno atributivo y no exactamente como reflexivo, ya que el ámbito de correferencia es apenas parcial.
} 


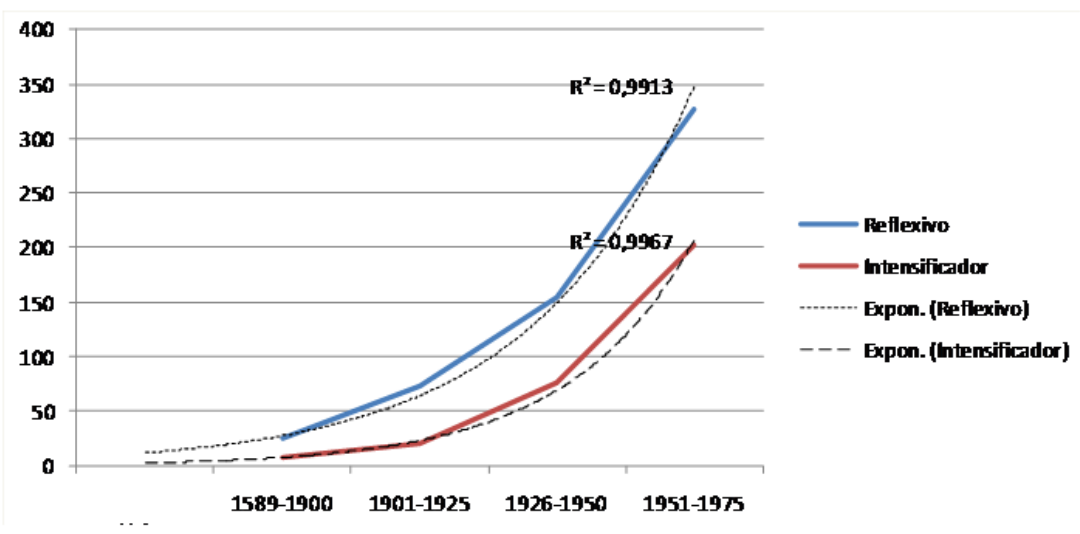

Gráfico 1: Distribución diacrónica de valores según la cantidad de textos.

Como se deduce del gráfico, el crecimiento es intenso para ambos valores. Si bien la tendencia que mejor describe estos casos es la polinómica, pues refleja las posibles fluctuaciones de ambas líneas, el crecimiento puede describirse perfectamente bien como exponencial, tal como se refleja con las líneas punteadas, lo cual pone en evidencia que los datos aumentan a intervalos cada vez mayores.

$\mathrm{Al}$ analizar las tasas de crecimiento de ambos valores, pero especialmente la del valor reflexivo, ciertamente se reconoce el auge ocurrido desde inicios del siglo XX, lo cual seguramente pueda explicarse con diversos factores: el mayor desarrollo industrial y su impacto en el vocabulario técnico, la mayor difusión de textos escritos con fines específicos, etc. (véase GarcíaMedall 1993). Sin embargo, el valor de intensificación posee una tasa de crecimiento mucho más alta de la que alguna vez alcanzó el valor reflexivo dentro del español con una tasa de crecimiento de un 194\% (1951-1975) versus un $143 \%$ (1926-1950):

\begin{tabular}{|l|c|c|c|c|}
\hline Periodo / & \multicolumn{2}{|c|}{ Reflexivo } & \multicolumn{2}{c|}{ Intensificador } \\
\hline $1589-1900$ & 25 & & 8 & \\
\hline $1901-1925$ & 73 & $192 \%$ & 21 & $163 \%$ \\
\hline $1926-1950$ & 155 & $112 \%$ & 76 & $262 \%$ \\
\hline $1951-1975$ & 327 & $111 \%$ & 202 & $166 \%$ \\
\hline
\end{tabular}

Tabla 2: Tasas de crecimiento según número de textos 
Respecto de la distinción según clases de palabras, se puede ver que la tendencia reflexiva sigue predominando en las cantidades absolutas en cada caso. Lo interesante, sin embargo, es que de forma transversal en las tres categorías gramaticales analizadas se repite el comportamiento de crecimiento de aparición, de forma que un valor del prefijo aumenta aproximadamente en la medida en que aumenta el otro:

\begin{tabular}{|l|c|c|c|c|c|c|c|}
\cline { 2 - 8 } \multicolumn{1}{c|}{} & \multicolumn{2}{c|}{ Sustantivos } & \multicolumn{2}{c|}{ Adjetivos } & \multicolumn{2}{c|}{ Verbos } & Total \\
\cline { 2 - 8 } \multicolumn{1}{c|}{} & Int. & Refl. & Int. & Refl. & Int. & Refl. & \\
\hline $1589-1900$ & 2 & 12 & 5 & 13 & 1 & 0 & 33 \\
\hline $1901-1925$ & 16 & 48 & 4 & 21 & 0 & 4 & 93 \\
\hline $1926-1950$ & 46 & 115 & 20 & 25 & 10 & 14 & 230 \\
\hline $1951-1975$ & 114 & 216 & 62 & 51 & 24 & 59 & 526 \\
\hline Totales & 178 & 391 & 91 & 110 & 35 & 77 & \\
\hline & \multicolumn{3}{|c|}{569} & \multicolumn{3}{c|}{201} & \multicolumn{2}{c|}{112} & $882^{7}$ \\
\hline
\end{tabular}

Tabla 3: Distribución de valores de auto- por periodo cronológico según clases de palabra

Evidentemente, al distribuir según clases de palabras, aparecen algunas diferencias, aunque sigue siendo notorio que en ningún caso el valor reflexivo aumenta de manera desmesurada con respecto al intensificador.

\section{DISCUSIÓN}

A partir de los resultados obtenidos, es posible hacer varias observaciones. La primera de ellas es el mencionado auge que se produce a partir del siglo $\mathrm{XX}$ en la productividad general de este prefijo, aunque por supuesto es difícil explicar este aumento mediante argumentos únicamente internos y no relacionados con una cada vez mayor disposición de datos. Es importante resaltar que en la búsqueda inicial de esta investigación se encontraron muchas ocurrencias durante los siglos anteriores que se descartaron por tratarse no del prefijo derivativo sino del tema auto-, como en automático.

\footnotetext{
7 Los cinco casos restantes en el total de 887 corresponden a adverbios no incluidos en esta tabla por ser estadísticamente poco significativos.
} 
Esto lleva a pensar que tal vez es posible ver un cambio diacrónico desde casos puramente cultos hacia los más innovadores de derivación a partir de este período. En este sentido, es interesante que precisamente la ocurrencia más antigua de los casos analizados sea autobiográfico que, si bien se puede considerar perfectamente como un derivado, ya que biográfico corresponde a un lexema del español, este último no deja de ser un cultismo. Ahora bien, una vez que el prefijo se establece como constituyente derivativo, se puede ver que es ampliamente productivo, tal como lo demuestra el hecho de que nuestro segundo registro sea autodeclarar (1699), con un valor claramente de intensificación y en la variante no pronominal. Entonces si bien, como afirma García-Medall (1993), las formas más antiguas corresponden a tecnicismos (esencialmente formaciones a partir del tema), una vez en el ámbito de la derivación, el prefijo auto- es muy rico y no se restringe al vocabulario técnico.

La segunda observación tiene que ver con las tendencias de crecimiento: ambos valores se desarrollan de manera paralela y no uno en detrimento del otro. Es importante notar que, si bien el aumento continuo puede explicarse por un incremento del número de textos en la base de datos, esto no justifica ni interfiere en el resultado de un desarrollo exponencial para ambos valores. Por lo tanto, como se planteó al comienzo de este trabajo, no podemos considerar este desarrollo como uno que propicie el rasgo secundario de un valor con respecto al otro. Sí se habría podido aceptar, en cambio, si la tendencia de crecimiento hubiera sido mayor en los reflexivos que en los intensificadores.

Por otro lado, esta homogeneidad de crecimiento se confirma también en la distribución de clases de palabras. Si el valor de intensificación estuviera limitado a alguna de las clases de palabras, también se podría pensar en algún tipo de rasgo secundario. Sin embargo, la alta productividad en todas las clases permite afirmar que se trata de un valor bastante natural. Es interesante señalar que, a diferencia de sustantivos y verbos, los adjetivos con valor de intensificación terminan en una distribución bastante pareja con respecto a los valores reflexivos. Esto podría relacionarse con algún grado de ambigüedad, presente en esta clase de palabras.

En definitiva, parece posible pensar que el valor no reflexivo no es necesariamente secundario con respecto al reflexivo, al menos en lo que refiere al origen y al desarrollo diacrónico, ya que ambos valores presentan un desarrollo sostenido a lo largo de los años. Así, podemos afirmar que los intensificadores se encuentran cuantitativamente por debajo de los reflexivos, con los cuales comparten el uso de constituyentes formalmente idénticos, pero no que sean realmente secundarios a lo largo de la historia del español. Como hipótesis (investigación que se encuentra en desarrollo al momento de la publicación del presente artículo), se puede proponer que, en todo caso, el 
cambio que tiene lugar en la historia del español es, tal como esperaríamos, el de un tema a un prefijo, es decir, un fenómeno de gramaticalización. Ahora bien, si quisiéramos intentar relacionar el desarrollo de auto- con la posible evolución de intensificadores a reflexivos, tal como ocurre en el ámbito verbal de muchas lenguas del mundo, deberíamos tener en cuenta un margen más amplio: el paso del latín al español, pues la mayoría de estas formaciones en latín (casi todos préstamos del griego y muy pocos calcos) son no reflexivas, como se ve en autocrator, automatus, autopator, autogenitor, automatarius. De esta manera, el fenómeno de formación de palabras visto aquí vendría a confirmar esa misma direccionalidad de cambio.

\section{CONCLUSIONES}

A modo de cierre, se puede concluir, en primer lugar, que si bien no se discute que desde una perspectiva histórica los valores de intensificación aparecen en menor cantidad que los reflexivos (lo que sí podría permitir hablar de un uso cuantitativamente secundario), no se encontró un periodo en el que el reflexivo fuera el único valor, por lo que no se puede afirmar que el valor de intensificación haya surgido de manera secundaria en términos diacrónicos. Por el contrario, ambos valores, que coexisten desde el comienzo de la forma prefijal, se van desarrollando gradual y paralelamente.

Por otro lado, si bien un análisis sincrónico podría llegar a demostrar que desde una perspectiva cognitiva los hablantes tienden a considerar hoy el valor reflexivo como primordial, es necesario preguntarse hasta qué punto esta percepción no se relaciona únicamente con una etiqueta, ya que también parece evidente la estrecha relación y una confluencia de ambos valores. En el análisis diacrónico aplicado a esta investigación, muy especialmente en el último periodo estudiado, se puso en evidencia la necesidad de considerar el contexto para poder determinar el tipo de valor en cada caso. Esto nos lleva a pensar que, de haber analizado varias de las formaciones de manera aislada y considerando únicamente los morfemas constituyentes, posiblemente habríamos caído en un prejuicio respecto de las categorías.

En segundo lugar, esperamos que este trabajo pueda representar un modesto aporte a la investigación teórica, en tanto el caso presentado no invalida la propuesta translingüística establecida acerca de una direccionalidad del cambio desde intensificadores hacia reflexivos en lo relativo a las formaciones nominales, pues se confirmarían direccionalidades de cambio propuestas previamente. Por último, y en relación con lo anterior, el desarrollo de estas formaciones permite poner en relación los cambios ocurridos en la formación de palabras y en constituyentes de las cláusulas 
verbales, pues incluso en casos que claramente no son deverbativos, la presencia de ciertos prefijos como auto- pueden establecer una determinada valencia, con lo que se puede equiparar ambos desarrollos, tanto sincrónica como diacrónicamente.

\section{REFERENCIAS BIBLIOGRÁFICAS}

Bosque, Ignacio y Violeta Demonte (eds.). 1999. Gramática descriptiva de la lengua española. Madrid: Espasa.

Faltz, Leonard. 1985 [1977]. Reflexivization: A Study in Universal Syntax. Nueva York: Garland.

Felí, Elena. 2005. Los sustantivos formados con el prefijo auto- en español: descripción y análisis. Verba 32: 331-350.

2003. Morfología derivativa y semántica léxica: la prefijación de auto-, co- e interMadrid: Ediciones de la Universidad Autónoma de Madrid.

García-Medall, Joaquín. 1993. Bibliografía comentada sobre los estudios de la prefijación en español. Thesaurus 3 (XLVIII): 556-613.

Givón, Talmy. 2001. Syntax: An Introduction. Vol. 1. Amsterdam: John Benjamins.

GONZÁlez, CARLOS. 2006.Las construcciones no reflexivas con "se": una propuesta desde la Gramática del Papel y la Referencia. Tesis doctoral. Universidad Complutense de Madrid.

Haspelmath, Martin. 2008. Parametric versus functional explanations of syntactic universals. En Theresa Biberauer (ed.). The limits of syntactic variation, pp. 75-107. Amsterdam: Benjamins.

HeINe, Bernd. 2005. On reflexive forms in creoles. Lingua 115 (3): 201-257.

Heine, Bernd y Tania Kuteva. 2002. World lexicon of grammaticalization. Cambridge: Cambridge University Press.

KÖNIG, EKKEHARD. 2011. Reflexive nominal compounds. Studies in Language 35 (1): 112-127.

KöNIG, EkKeHARD y Peter Siemund. 2000. Intensifiers and reflexives - a typological perspective. En Frajzyngier, Zigmunt y Traci Curl (eds.). Reflexives: Forms and Function, pp. 41-74. Amsterdam: John Benjamins.

König, Ekkehard, Peter Siemund y Stephan Töpper. 2013. Intensifiers and Reflexive Pronouns. En Matthew Dryer y Martin Haspelmath (eds.). The World Atlas of Language Structures [en línea]. Disponible en: http://wals.info/chapter/47 [Consulta 09/05/2016].

Lang, Mervyn. 1990. Formación de palabras en español. Madrid: Cátedra.

Maldonado, Ricardo. 1999. A media voz. Problemas conceptuales del clítico se. México: Universidad Nacional Autónoma de México.

Mutz, Katrin. 2003. Le parole complesse in 'auto' nell'italiano di oggi. En Maraschio, N. y T. Poggi Salani (eds.). Italia linguistica anno Mille. Italia linguistica anno Duemila. Atti del XXXIV congresso internazionale di studi della SLI. Firenze, 19-21 novembre 2000, pp. 649-664. Roma: Bulzoni.

Real Academia Española. s/a. Corpus Diacrónico del Español (CORDE) [en línea]. Disponible en http://corpus.rae.es/cordenet.html [Consulta 15/05/2016].

2010. Manual de la nueva gramática de la lengua española. Madrid: Espasa.

Schladt, Matthias. 2000. The typology and grammaticalization of reflexives. En Zigmunt Frajzyngier y Traci Curl (eds.). Reflexives: Forms and Functions, pp. 103-124. Amsterdam: John Benjamins.

Van Valin, Robert. 2001. An introduction to syntax.Cambridge: Cambridge University Press. Varela, Soledad. 2005. Morfología léxica: la formación de palabras. Madrid: Gredos. 